See discussions, stats, and author profiles for this publication at: https://www.researchgate.net/publication/321630898

\title{
A comparison of DSM-5 and ICD-11 PTSD prevalence, comorbidity and disability: An analysis of the Ukrainian Internally Displaced Person's Mental Health Survey
}

Article in Acta Psychiatrica Scandinavica · December 2017

DOI: $10.1111 /$ acps. 12840

CITATIONS

23

8 authors, including:

Mark Shevlin

Ulster University

377 PUBLICATIONS 8,771 CITATIONS

SEE PROFILE

-

Frédérique Vallières

Trinity College Dublin

64 PUBLICATIONS 387 CITATIONS

SEE PROFILE
READS

336

6 Philip Hyland

13 National University of Ireland, Maynooth

181 PUBLICATIONS 2,241 CITATIONS

SEE PROFILE

Jonathan I Bisson

Cardiff University

202 PUBLICATIONS 9,080 CITATIONS

SEE PROFILE

Some of the authors of this publication are also working on these related projects:

RAPID - Pragmatic RAndomised controlled trial of a Trauma-Focused Guided Self Help Programme versus InDividual Trauma-Focused Cognitive Behavioural Therapy for Post-Traumatic Stress Disorder (RAPID) View project

Developing Institutional Mechanisms for Addictology Education in Georgia View project 


\section{Acta Psychiatrica Scandinavica}

\section{A Comparison of DSM-5 and ICD-11 PTSD Prevalence, Comorbidity, and Disability.}

\begin{tabular}{|r|l|}
\hline Journal: & Acta Psychiatrica Scandinavica \\
\hline Manuscript ID & ACP-2017-6789.R2 \\
\hline Danuscript Type: & Original Article \\
\hline Complete List of Authors: & $\begin{array}{l}\text { Shevlin, Mark; Ulster, Psychology; } \\
\text { Hyland, Philip; National College of Ireland, School of Business } \\
\text { Vallières, Frédérique ; University of Dublin Trinity College, Global Health } \\
\text { Bisson, Jonathan I; Cardiff University, School of Medicine } \\
\text { Makhashvili, Nino; Ilia State University, Global Initiative on Psychiatry } \\
\text { Javakhishvili, Jana ; Ilia State University, Tbilisi, Georgia., Global Initiative } \\
\text { on Psychiatry } \\
\text { Shpiker, Marina; Kiev International Institute of Sociology , Sociology } \\
\text { Roberts, Bayard; London School of Hygiene and Tropical Medicine, The } \\
\text { Centre for Health and Social Change }\end{array}$ \\
\hline Keywords: & PTSD, Trauma, Classification, Comorbidity, Diagnosis \\
\hline \multicolumn{2}{|c}{} \\
\hline
\end{tabular}


A Comparison of DSM-5 and ICD-11 PTSD Prevalence, Comorbidity, and Disability: An Analysis of the Ukrainian Internally Displaced Person's Mental Health Survey Mark Shevlin ${ }^{1}$, Philip Hyland ${ }^{2,3}$, Frederique Vallières ${ }^{3}$, Jon Bisson ${ }^{4}$, Nino Makhashvili ${ }^{5}$, Jana Javakhishvili ${ }^{5}$, Marina Shpiker ${ }^{6}$, Bayard Roberts ${ }^{7}$

${ }^{1}$ Psychology Research Institute, Ulster University, Londonderry, Northern Ireland.

${ }^{2}$ School of Business, National College of Ireland, Dublin, Ireland.

${ }^{3}$ Centre for Global Health, University of Dublin, Trinity College, Dublin, Ireland.

${ }^{4}$ Division of Psychological Medicine and Clinical Neurosciences, Cardiff University, Cardiff, Wales.

${ }^{5}$ Global Initiative on Psychiatry, Tbilisi, Georgia; Ilia State University, Tbilisi, Georgia.

${ }^{6}$ Kiev International Institute of Sociology (KIIS) Kiev, Ukraine.

${ }^{7}$ ECOHOST-The Centre for Health and Social Change, London School of Hygiene and Tropical Medicine, London, United Kingdom. 


\begin{abstract}
Objective: Recently, the American Psychiatric Association (DSM-5) and the World Health Organization (ICD-11) have both revised their formulation of posttraumatic stress disorder (PTSD). The primary aim of this study was to compare DSM-5 and ICD-11 PTSD prevalence and comorbidity rates, as well as the level of disability associated with each diagnosis. Method: This study was based on a representative sample of adult Ukrainian internally displaced persons (IDPs: $N=2,203$ ). PTSD prevalence was assessed using the PTSD Checklist for DSM-5 and the International Trauma Questionnaire (ICD-11). Anxiety and depression were measured using the Generalized Anxiety Disorder Scale and the Patient Health QuestionnaireDepression. Disability was measured using the WHO Disability Assessment
\end{abstract} Schedule 2.0.

Results: The prevalence of DSM-5 PTSD (27.4\%) was significantly higher than ICD11 PTSD (21.0\%), and PTSD rates for females were significantly higher using both criteria. ICD-11 PTSD was associated with significantly higher levels of disability and comorbidity.

Conclusion: The ICD-11 diagnosis of PTSD appears to be particularly well suited to identifying those with clinically relevant levels of disability.

Key words: Posttraumatic stress disorder (PTSD); DSM-5; ICD-11; Depression; Anxiety; Internally displaced persons. 


\section{Significant Outcomes}

- In a large representative sample of adult Ukrainian IDPs the prevalence of DSM-5 PTSD was $27.4 \%$ and $21.0 \%$ for ICD-11 PTSD.

- Higher levels of comorbidity with anxiety and depression were found for those who met the criteria for ICD-11 PTSD.

- Higher levels of disability were found for those who met the criteria for ICD-11 PTSD.

Limitations

- Self-reported, rather than clinician assessed, PTSD symptoms were used.

- Disability, as measured using the WHODAS 2.0, was assessed only for the previous 30 days.

- The findings may not generalize to IDP populations in culturally distinct contexts. 
A Comparison of DSM-5 and ICD-11 PTSD Prevalence, Comorbidity, and Disability: An Analysis of the Ukrainian Internally Displaced Person's Mental Health Survey

The United Nations High Commissioner for Refugees (UNHCR) reported that by the end of $2016,65.6$ million people worldwide were forcibly displaced due to conflict, violence, or human rights violations ${ }^{1}$; a record high figure. Of these people, 40.3 million were forcibly displaced within their own country meaning that internally displaced people (IDPs) constitute approximately two-thirds of all displaced people worldwide. In Ukraine, specifically, there are currently 1.8 million IDPs, equating to a rate of 100 per 1,000 of the population ${ }^{1}$. IDPs are commonly exposed to multiple traumatic life events including war, sexual and physical violence, torture, and witnessing death and extreme human suffering ${ }^{1}$. Recognising the immensely deleterious mental health effects of forced displacement for such a large proportion of the world's population, the World Bank and the World Health Organization (WHO) recently issued a joint call for the prioritization of mental health as a key component of the global health agenda ${ }^{2}$.

The American Psychiatric Association (APA) ${ }^{3}$ and the $\mathrm{WHO}^{4}$ have recently revised their formulation of posttraumatic stress disorder (PTSD); a psychiatric disorder commonly observed in forcibly displaced people ${ }^{5}$. The fifth edition of the APA's Diagnostic and Statistical Manual of Mental Disorders (DSM-5) includes 20 symptoms of PTSD that are organised under four symptom clusters; intrusions, avoidance, negative alternations in cognitions and mood (NACM), and alternations in arousal and reactivity (AR). A diagnosis of PTSD requires exposure to a Criterion A stressor and the endorsement of at least one intrusion symptom, one avoidance symptom, two NACM symptoms, and two AR symptoms. Additionally, these symptoms must be associated with functional impairment, and be present for more 
than one month. The proposed model of PTSD set forth for the $11^{\text {th }}$ version of the International Classification of Diseases (ICD-11) published by the WHO includes six symptoms organised under three symptom clusters; re-experiencing of the traumatic event(s) in the here and now (Re), avoidance of traumatic reminders (Av), and a sense of current threat (Th). A diagnosis of PTSD requires exposure to an extreme stressor and the endorsement of at least one symptom from each cluster. Additionally, symptoms must be associated with functional impairment, and be present for several weeks.

The ICD-11 proposals for a reduced set of PTSD symptoms were based on the desire to (1) simplify the assessment of PTSD to enhance clinical utility, particularly in low-resourced and/or humanitarian crisis contexts, (2) reduce the high level of symptom profile heterogeneity, and (3) reduce the co-morbidity with other disorders ${ }^{4}$. The simplification of assessment and diagnosis is clearly achieved with a much-reduced symptom set. Galatzer-Levy and Bryant ${ }^{5}$ showed that for DSM-5 PTSD there are 636,120 different possible symptom combinations for a diagnosis. Applying the same methods to ICD-11 PTSD, there are only 27 possible symptom combinations for a diagnosis. Whether the ICD-11 model of PTSD reduces comorbidity with other disorders is unclear due to the limited number of studies. O'Donnell et al. ${ }^{6}$ reported that in a sample of 953 injury patients, DSM-5 PTSD was associated with an $11 \%$ higher comorbidity rate with depression as compared to ICD-11, although this difference was not statistically significant. Hafstad et al. ${ }^{7}$ reported that for parents of young survivors of the 2011 Norway attacks $(n=451)$, ICD-11 PTSD was associated with higher levels of anxiety and depression comorbidity compared to DSM-5 PTSD, but this difference was not significant. 
Studies that have examined differences in prevalence rates between the two diagnostic criteria have been somewhat mixed. Significantly higher rates of DSM-5 PTSD have been reported amongst physical injury patients in Australia ${ }^{6}$, adult survivors of childhood sexual abuse in Denmark ${ }^{8}$, community and veteran samples in the United States ${ }^{9}$, motor vehicle and incest survivors in Denmark ${ }^{10}$, and treatment-seeking patients in Scotland ${ }^{11}$. In contrast, Stein et al. ${ }^{12}$ found no difference between DSM-5 and ICD-11 PTSD based on pooled cross-national data from 23,936 community participants. Likewise, Hansen et al. ${ }^{10}$ reported no significant differences in PTSD prevalence in five Danish samples (bereaved parents, paraplegics, physical assault victims, sexual assault victims, and help-seeking trauma patients).

The main limitation of existing research comparing DSM-5 and ICD-11 PTSD prevalence and comorbidity is that, with the exception of one study ${ }^{11}$, a standardised and validated measure of ICD-11 PTSD has not been utilized. To date, ICD-11 PTSD has generally been assessed using a subset of symptom indicators originally developed to measure DSM-based symptoms (DSM-IV or DSM-5). Given that the specific symptom content of ICD-11 PTSD does not correspond precisely to the DSM-based symptoms, the existing estimates of prevalence and comorbidity across the two criteria may be misleading. A disorder-specific measure of ICD-11 PTSD (and Complex PTSD), the International Trauma Questionnaire (ITQ $)^{13}$, was recently developed and validated ${ }^{14,15}$. It is important that any assessment of prevalence rates and comorbidity of ICD-11 PTSD be based on an instrument that accurately measures the ICD-11 PTSD symptom content, and therefore acknowledges the important differences between the ICD-11 and DSM-5 symptom criteria. In particular, the ICD-11 emphasises re-experiencing of the traumatic event 'in the here and now' 
as an essential feature of PTSD; a distinction that is often not captured by proxymeasures designed for DSM-IV or DSM-5 PTSD. Several studies have suggested that differences between DSM-5 and ICD-11 PTSD diagnostic rates are attributable to differences in the proportion of individuals meeting this criterion ${ }^{7,8}$. Another limitation of the extant research is the use of non-representative, opportunistic samples; to date there has been no evaluation of ICD-11 and DSM-5 prevalence and co-morbidity rates based on large probability-based samples; or amongst IDPs.

The primary aim of this study was to compare ICD-11 (using the ITQ) and DSM-5 (using the PCL-5) PTSD prevalence and comorbidity rates, in a large, representative sample of Ukrainian adult IDPs. On the basis that (a) the current study is the first to assess prevalence and comorbidity rates amongst an IDP sample; (b) the scant evidence regarding comorbidity across the two criteria; and (c) the inconsistent findings regarding prevalence estimates of DSM-5 versus ICD-11 PTSD, no formal hypotheses were formulated. Rather, we investigated four study aims:

1. To determine the prevalence rates, and gender differences, of DSM-5 and ICD-11 PTSD amongst a representative sample of adult Ukrainian IDPs. Additionally, we sought to identity individual's that represented 'unique' cases of DSM-5 PTSD (i.e., those that meet the diagnostic criteria for DSM-5 but not ICD-11 PTSD) and 'unique' cases of ICD-11 PTSD (i.e., those that meet the diagnostic criteria for ICD-11 but not DSM-5 PTSD).

2. To determine if there are differences in levels of disability amongst IDPs that meet diagnostic status for DSM-5 and ICD-11 PTSD. Differences in levels of disability were also compared across 'unique' DSM-5 and ICD-11 PTSD cases. 
3. To determine the degree of comorbidity for ICD-11 and DSM-5 PTSD with Generalized Anxiety Disorder (GAD), Major Depressive Disorder (MDD), and combined GAD and MDD. Comorbidity rates for 'unique' ICD-11 and DSM-5 PTSD cases were also assessed.

4. To determine if specific types of trauma are differentially associated with ICD-11 and DSM-5 PTSD. The association between specific trauma types and a 'unique' ICD-11 PTSD diagnosis, as compared to a 'unique' DSM-5 PTSD diagnosis, was also assessed.

\section{METHODS}

\section{Participants}

Data comes from the "Internally Displaced Person's Mental Health" survey that was carried out from March to May 2016 all over Ukraine excluding occupied territories ${ }^{17}$. The survey covered all oblasts of Ukraine and 74 settlements (mainly urban). Time-Location Sampling was chosen as a probabilistic method to recruit hard-to-reach and migrant populations ${ }^{17}$. In total, 121 unique locations were used for recruitment during the survey: $33.0 \%$ from collective centers, $31.0 \%$ from NGOs that work with IDPs, $6.0 \%$ from state institutions, $24.0 \%$ were recruited with the help of another person (informant), and $6.0 \%$ were reached by other means. A weighting variable was calculated to correct the regional structure of the sample in accordance with official statistics and was applied for all analyses.

The sample $(\mathrm{N}=2,198)$ includes male and female IDPs $(91.8 \%$ had official IDP status with the UNHCR) who live both in institutional and non-institutional settings on the territories controlled by the Ukrainian government. Following agreed definitions for IDPs ${ }^{18}$, a person in the current study was considered an IDP if they 
answered 'yes' to the screening question that they had been forced to flee their home because of conflict and were currently living away from their home. Exclusion criteria included people deemed under the influence of alcohol or drugs, and those with severe intellectual or mental impairment, at the time of the survey. Data collectors were trained in the identification of these predetermined exclusion criteria which related to criteria of understanding, expression, communication, and behaviour. The mean time since displacement was 17.49 months $(S D=4.49)$. The sample included 1496 (68.1\%) females, and the mean age was 45 years (SD = 16.99). The majority of participants reported being married or co-habiting $(52.7 \%)$, $20.2 \%$ were single, $14.3 \%$ were divorced, and $12.8 \%$ were widowed. Most participants had completed higher education (35.9\%) or secondary technical education $(29.5 \%)$ with the remaining having lower levels of educational attainment. Most participants who were working were in regular paid work $(22.4 \%)$ with others in irregular paid work (9.9\%) or self-employed (2.8\%); $28.9 \%$ were retired due to old age or invalidity and $17.9 \%$ were unemployed and seeking work. The remaining participants $(18.1 \%)$ were doing voluntary work, students, homemakers, or on maternity leave.

The questionnaires were completed through face-to-face interviews in either Ukrainian or Russian by trained enumerators from the Kiev International Institute of Sociology (KIIS) in a private space chosen by the respondent. Before administering the questionnaire, each respondent listened to the explanations about the aim of the survey and terms of participation. In addition, the participant received an information sheet and consent form and then gave either written or verbal consent. Ethical approval was provided by the KIIS Institutional Review Board. All team leaders of regional groups of interviewers were instructed and trained before the survey, and 
the team leaders provided the training to their teams of experienced data collectors. The response rate of IDPs was around $90 \%$ in the whole sample.

\section{Measures}

\section{ICD-11 PTSD}

The ITQ ${ }^{13}$ is a preliminary stage, self-report measure of ICD-11 PTSD (and Complex PTSD) symptomatology. Six items represent the three clusters of PTSD $(\mathrm{Re}, \mathrm{Av}, \mathrm{Th})$ using two items each. An additional three items measure functional impairment associated with these symptoms (e.g., impairment in (1) social, (2) occupational, and (3) parenting or other important activities). All items are answered on a five-point Likert scale anchored by "Not at all” (0) and "Extremely" (4). Diagnostic criteria require a score of $\geq 2$ ("Moderately") for at least one symptom from each cluster, along with endorsement of at least one functional impairment item. Amongst the current IDP sample the reliability of the total scale $(\alpha=.89)$ and the $\operatorname{Re}(\alpha=.86), \operatorname{Av}(\alpha=.75)$, and Th $(\alpha=.81)$ subscales were good.

\section{DSM-5 PTSD}

The PCL- $5^{19}$ is a 20 -item, self-report measure capturing each DSM-5 PTSD symptom. Participants respond using the same 5-point Likert scale as the ITQ. Diagnostic criteria require a score of $\geq 2$ ("Moderately") for at least one intrusion symptom, one avoidance symptom, two NACM symptoms, and two arousal symptoms. Endorsement of at least one functional impairment item is also required (the PCL-5 and ITQ use the same functional impairment items). The PCL-5 has demonstrated acceptable reliability and validity in previous studies ${ }^{20}$. Amongst the 
current sample, the reliability of the total scale $(\alpha=.96)$ and the intrusions $(\alpha=.89)$, avoidance $(\alpha=.88)$, NACM $(\alpha=.90)$, and AR $(\alpha=.89)$ subscales were good.

\section{Lifetime Traumatic Exposure}

The Life Events Checklist (LEC) ${ }^{21}$ is a 17-item, self-report measure screening for lifetime traumatic exposures. The LEC assesses lifetime exposure to 16 traumas plus one open question for respondents to indicate any other traumatic event not listed. The overwhelming majority of respondents indicated exposure to at least one traumatic life event $(93.3 \%, n=2051)$ and the most commonly reported trauma was exposure to war or combat $(71.3 \%, n=1566)$. For each item, the respondent checks whether the event 'Happened to me' (1), 'Witnessed it happening to somebody else' (2), 'Learned about it happening to someone close to me' (3), 'Part of my job' (4), 'Not sure it applies' (5), 'Doesn't apply to my experience' (6). The scores were recoded into binary variables with 'Happened to me' responses being coded as 1 and all other responses coded as 0 , except for the 'Sudden violent death' and 'Sudden accidental death' items which were coded 1 for 'Witnessed it happening to somebody else' and all other responses coded as 0.

\section{Measures of Psychiatric Comorbidity}

The Generalized Anxiety Disorder Scale (GAD-7) $)^{22}$ is a 7 -item, self-report measure assessing each DSM-IV symptom of GAD. The instructions state, "Over the last 2 weeks, how often have you been bothered by any of the following problems?" and participants respond using a 4-point Likert scale ("Not at all" (0) to "nearly every day" (3)). Total scores on the GAD-7 range from 0 to 21 . Scores $\geq 10$ are used as a cut-off to identify diagnosis of $\mathrm{GAD}^{23}$. The reliability of the scale in this sample was high $(\alpha=.93)$. 
The Patient Health Questionnaire-Depression $(\mathrm{PHQ}-9)^{24}$ is a 9-item, selfreport measure assessing each DSM-IV symptom of MDD. Instructions and response options are the same as the GAD-7. Total scores range from 0 to 27 . Scores $\geq 10$ are used as a cut-off to identify diagnosis of $\mathrm{MDD}^{26}$. The reliability of the scale in this sample was high $(\alpha=.91)$.

\section{Disability}

The WHO Disability Assessment Schedule 2.0 (WHODAS 2.0$)^{26}$ is a 12 -item self-report measure that assesses activity limitations and participation restrictions in six domains (Understanding and communicating, Mobility, Self-care, Social functioning, Household activities, Community activities). The instructions state, "In the last 30 days how much difficulty did you have in...", and participants respond to each item using a 5-point Likert scale ranging from "None" (0) to "Extreme or cannot do" (4). Total scores range from 0 to 48 with higher scores reflecting greater levels of disability. No agreed upon cut-off score exists for the WHODAS 2.0, however scores $\geq 10$ have been shown to include the top $10 \%$ of the population distribution of WHODAS 2.0 scores, and therefore likely to reflect those with clinically relevant levels of disability ${ }^{27}$. Consistent with previous PTSD-based studies ${ }^{7}$, a score $\geq 10$ was used in the current study to identify individuals with 'clinically significant levels of disability'. The reliability of the scale in this sample was high $(\alpha=.94)$.

The survey questionnaire was largely developed in English and then underwent a thorough adaptation and translation process into Ukrainian and Russian based on best practice procedures to help ensure reliability, validity and appropriateness with the study population. This included: (i) translation from English into Ukrainian and Russian using professional translators, with translations reviewed 
by mental health experts individually and then as a group for cultural relevance, content and concept consistency, clarity, and understanding; (ii) a back-translation to check for accuracy, consistency, and equivalence, with adjustments made accordingly; and (iii) piloting and field testing ${ }^{a 1}$ to refine the instruments further ${ }^{28}$. Ukrainian and Russian versions of the PCL-5, PHQ-9, and LEC-5 instruments were used as they already existed but were still subject to the back-translation and expert discussion.

\section{Analysis}

The prevalence rates were estimated using the weighted frequencies (and percentages) with $95 \%$ confidence intervals, and group comparisons were conducted using the exact McNemar binomial test. Differences in percentages were expressed as 'percentage change' $\left(\left(\%_{1}-\%_{2}\right) /\left(\%_{1}\right) \times 100\right)$. The reliability of all selfreport scales were assessed using Cronbach's alpha. The associations between trauma exposure and diagnostic status were estimated using binary logistic regression.

\section{RESULTS}

\section{Prevalence Rates}

\footnotetext{
${ }^{a 1}$ The piloting was conducted with approximately 30 IDPs in Kiev who were not included in the main survey conducted later on. The piloting sought to assess the (1) clarity, comprehension, comprehensiveness of the questionnaire, (2) understanding of the consent procedures, (3) feasibility (e.g. questionnaire administration time, respondent willingness to participate), (4) potential for respondent burden and distress, (5) socio-cultural appropriateness, and (6) formatting of the questionnaire (e.g. skip patterns, ordering of the questions). Any key findings were noted by data collectors. They also asked participants about the survey instrument and any comments they had on it (based on the criteria noted above). The findings were then shared with the management team and discussed and alterations made where appropriate.
} 
The prevalence of DSM-5 PTSD was $27.4 \%(95 \% \mathrm{Cl}=25.52-29.33 \%)$, while the prevalence of ICD-11 PTSD was $21.0 \%(95 \% \mathrm{CI}=19.18-22.65 \%)$; representing an increase of $30.5 \%$ in diagnostic rates under DSM-5 (see Table 1). This difference in diagnostic rates according to the two criteria was statistically significant (exact binomial test: $p<.001$ ). There were a larger number of 'unique' DSM-5 PTSD cases $(9.6 \%, n=197)$ than 'unique' ICD-11 PTSD cases $(3.5 \%, n=$ 71). Additionally, $17.6 \%(n=362)$ of IDPs met the diagnostic criteria for both DSM-5 and ICD-11 PTSD; a level of diagnostic agreement that was considered to be 'substantial' ${ }^{30}(\mathrm{k}=.64, \mathrm{SE}=.02, \mathrm{p}<.001)$.

DSM-5 PTSD rates were significantly higher for females $(31.1 \% ; 95 \% \mathrm{Cl}=$ $29.05-33.92 \%)$ than males $(19.6 \% ; 95 \% \mathrm{Cl}=16.29-22.33 \%)$ representing an increase of $58.1 \%$. ICD-11 PTSD rates were also significantly higher for females $(24.6 \% ; 95 \% \mathrm{Cl}=22.57-27.11 \%)$ than males $(13.1 \% ; 95 \% \mathrm{Cl}=10.46-15.61 \%)$ representing an increase of $87.8 \%$ (see Table 1).

Table 1 here

The difference in PTSD prevalence across the two diagnostic systems is primarily due to variation in the proportion of IDPs that meet the respective 'Intrusions' (DSM-5: 71.5\%) and 'Re-experiencing' (ICD-11: 31.9\%) symptom criteria. The proportion of individuals meeting criteria for Avoidance (DSM-5 $=54.1 \%$, ICD-11 = 51.0\%), and Arousal (DSM-5: 39.1\%) and Sense of Threat (ICD-11: 40.2\%) were concordant.

\section{Disability and Comorbidity Rates}


Amongst those with a DSM-5 PTSD diagnosis, 48.5\% experienced clinically significant levels of disability, and $57.6 \%$ of those with an ICD-11 PTSD diagnosis experienced clinically significant levels of disability. 'Unique' ICD-11 PTSD was associated with significantly higher levels of disability compared to 'unique' DSM-5 PTSD (36.7\% v. $28.6 \%$, exact binomial test: $p<.001)$.

Based on the GAD-7, $16.1 \%$ of participants met the criteria for GAD, and based on the PHQ-9, $20.6 \%$ of the participants met the criteria for MDD; $10.8 \%$ met the criteria for both GAD and MDD. The rates of comorbidity of PTSD with GAD, MDD, and combined GAD and MDD, respectively, were all higher for ICD-11 PTSD than DSM-5 PTSD. For 'unique' cases of PTSD, comorbidity rates with GAD, and combined GAD and MDD, were significantly higher for ICD-11 PTSD, whereas comorbidity with MDD was significantly higher for DSM-5 PTSD (Table 2).

Table 2 here

\section{PTSD and Traumatic Exposure}

A series of bivariate binary logistic regression models were tested. Each trauma from the LEC was used as a predictor variable and the ICD-11 and DSM-5 diagnoses were used as criterion variables (see Table 3). Most traumatic experiences were significantly related to a DSM-5 PTSD diagnosis, except for 'exposure to a war-zone' and 'captivity'. The largest effects were for 'serious injury, harm, or death you caused to someone else' $(O R=3.00)$, 'sexual assault' $(O R=$ 2.95), and 'life-threatening illness or injury' (OR = 2.59). Most traumatic experiences were also significantly related to ICD-11 PTSD, except for 'captivity'. The largest effects were for 'sexual assault' $(O R=3.66)$, 'other unwanted or uncomfortable 
sexual experience' (OR = 3.28), and 'serious injury, harm, or death you caused to someone else' (OR = 2.80).

Finally, a series of bivariate binary logistic regression analyses were conducted to determine the association between each trauma from the LEC and a 'unique' ICD-11 PTSD diagnosis (the 'unique' DSM-5 PTSD diagnosis group were used as the reference category for these analyses). As detailed in Table 3, one traumatic event, 'severe human suffering', was significantly related to an elevated risk of 'unique' ICD-11 PTSD (OR = 1.76). Exposure to a 'transportation accident' was significantly related to a reduced risk of 'unique' ICD-11 PTSD (OR = .22).

Table 3 here

\section{Discussion}

The primary objective of the current study was to provide the first methodologically rigorous assessment of the prevalence and comorbidity rates of ICD-11 and DSM-5 PTSD in a large, representative sample of internally displaced Ukrainian adults. The first aim of the study was to determine if prevalence rates of PTSD varied significantly across the two diagnostic systems and we found that DSM-5 was associated with $30.5 \%$ more diagnostic cases. Although the reported level of kapp (.64) is described as 'substantial' according to Cohen's criteria, it represents a level of agreement that is lower than expected for two measures that purportedly measure the same construct. More context specific interpretations of kappa have been proposed by $\mathrm{McHugh}^{30}$ who suggested that levels of agreement over .80 are more appropriate in clinical research. These findings are consistent with a number of studies that have reported significantly lower rates of PTSD for ICD-11 compared to DSM-5, $5^{7,8-11}$. Furthermore, as in prior studies ${ }^{7,8}$, the significantly lower 
rates of ICD-11 PTSD were largely attributable to fewer individuals satisfying the newly proposed 're-experiencing' criteria. The stipulation of re-experiencing the traumatic event in the here and now for ICD-11 may represent a less common psychological reaction as compared to other forms of re-experiencing that are included within the DSM-5 (e.g., becoming upset upon reminders of a trauma). The focus on present-moment re-experiencing may result in the ICD-11 diagnosis being more 'difficult' to attain, that is, a person requires a more intense and distressing reliving of the trauma before meeting the diagnostic criteria. Another possibility is that the broader nature of DSM-5 PTSD, with the inclusion of multiple non-trauma specific symptoms, can result in an 'easier' PTSD diagnosis. If one considers that the psychological response to trauma can be represented as an underlying continuum of distress severity, then the threshold for a DSM-5 diagnosis lies lower on the continuum than an ICD-11 diagnosis.

This conclusion is supported by the results of the association between PTSD status and disability. A significantly greater number of individuals who met diagnosis under ICD-11, compared to DSM-5, exhibited clinically meaningful levels of disability. The same pattern of results was also identified when unique cases of ICD-11 and DSM-5 PTSD were compared. Although a PTSD diagnosis according to both the DSM-5 and the ICD-11 identified a substantial number of people with high levels of impairment in daily functioning, it appears that the ICD-11 diagnosis, in particular, is sensitive at capturing individuals experiencing clinically significant levels of disability.

The study's second aim was to assess whether significant gender differences existed in PTSD prevalence for DSM-5 and ICD-11 PTSD. For the ICD-11 diagnosis, the rate for females was almost double that for males, and for DSM-5 the increase was approximately one third. This is consistent with the plethora of research that has 
identified significant gender differences ${ }^{31}$ but the difference in prevalence based on the DSM-5 was smaller than the 2:1 ratio that has been reported in population based studies $^{31}$.

Although PTSD prevalence was common amongst this sample of Ukrainian IDPs, irrespective of which diagnostic systems was selected, the level of diagnostic agreement between the two systems was quite low. Only $17.6 \%$ of IDPs were diagnosed with PTSD according to both the ICD-11 and DSM-5 diagnostic algorithms. A substantial number of IDPs qualified for a diagnosis of PTSD according to the DSM-5 but not the ICD-11 (9.6\%), while a smaller number qualified for a diagnosis of PTSD according to the ICD-11 but not the DSM-5 (3.5\%). This demonstrates that there are a considerable number of trauma-survivors who will only meet diagnostic status under one of the two available criteria. This circumstance has been highlighted as a challenge to the field of psychotraumatology as victims of trauma, and their caregivers, may be denied compensation and/or insurance coverage depending upon which diagnostic system is selected ${ }^{32}$. Regrettably, this set of affairs is likely to persist into the near future and until there is an agreed-upon diagnostic model of PTSD.

The third aim of this study was to assess the degree of comorbidity for ICD-11 and DSM-5 PTSD with GAD, MDD, and combined GAD and MDD. The ICD-11 proposals for a reduced symptom set related to 'core' PTSD symptoms were, in part, aimed at reducing the high levels of comorbidity that have been reported, particularly with other mood and anxiety disorders ${ }^{33}$. However, contrary to this proposition, significantly higher levels of comorbidity with GAD (7.9\%), MDD (6.5\%) and combined GAD and MDD (8.1\%) were associated with ICD-11 PTSD, not DSM-5 PTSD. An interesting pattern of comorbidity emerged when unique ICD-11 and 
DSM-5 PTSD cases were compared. The unique ICD-11 PTSD cases were associated with higher comorbidity rates for GAD and combined GAD and MDD, whereas unique cases of DSM-5 PTSD were associated with higher comorbidity rates for MDD. These findings suggest that ICD-11 PTSD shares more in common with anxiety disorders, while DSM-5 shares more in common with depressive disorders. These findings are congruent with theoretical proposals ${ }^{4}$ and prior empirical results ${ }^{6}$.

The high levels of comorbidity, and the specific patterns of comorbidity, are difficult to interpret within a traditional categorical model of psychopathology where a focus on unique symptoms rather than trans-diagnostic symptoms should reduce comorbidity. However, these findings do make sense within a dimensional model of psychopathology. Recently, Kotov et al. ${ }^{34}$ proposed the 'Hierarchical Taxonomy of Psychopathology' (HiTOP) model that is based on an abundance of empirical data indicating that psychiatric disorders cluster into meaningful superordinate latent factors (e.g., dimensions of 'Internalising', 'Externalising', and 'Thought Disorder') ${ }^{37}$. The HiTOP model considers diagnoses such as PTSD, GAD, and MDD as 'syndromes' occurring under the 'Distress' factor; a sub-factor subsumed by a superordinate 'Internalising' psychopathology dimension. This hierarchical model assumes a causal system similar to the factor analytic or 'effect' indicator model ${ }^{38}$ which posits downward causal effects. This means that increased levels of Internalising psychopathology will increase the likelihood of higher levels of the 'Distress' sub-factor which will consequently result in increased scores in all of its 'indicators' (i.e., syndromes) such PTSD, GAD, and MDD. In other words, under the assumption of a dimensional model of psychopathology, high levels of comorbidity between PTSD, GAD, and MDD are unavoidable given their shared relationship to 
an underlying latent variable (Internalizing). Furthermore, as in factor analytic models, when 'indicators' (e.g., PTSD) are measured with greater precision and less error (e.g., by focusing on 'core symptoms'), the observed covariation with other indicators (e.g., GAD, MDD) will inevitably increase. The objective of the revision to ICD-11 PTSD to reduce comorbidity with other 'Internalizing' disorders through a focus on core symptoms may therefore be unachievable given the assumptions associated with a categorical model of psychopathology.

There are two other factors that may help to explain the observed comorbidity rates. First, it is likely that exposure to traumatic stressors is an etiological risk factor not just for PTSD, but also for many other psychiatric conditions including depression and anxiety. As the ICD-11 model of PTSD reflects a more 'specific' disorder than DSM-5 PTSD, it is important that clinicians screen for other conditions such as GAD and MDD amongst trauma-exposed persons when adopting the use of the ICD-11 model of PTSD. Secondly, the current findings suggest that ICD-11 PTSD also represents a more 'severe' psychiatric disorder than DSM-5 PTSD. Consequently, an increased likelihood of diagnostic comorbidity associated with ICD-11 PTSD may be precisely what one should expect to observe.

The study's fourth objective sought to determine if there are specific types of trauma that are differentially associated with ICD-11 and DSM-5 PTSD. When examining the relative relationships between each trauma type and risk of DSM-5 or ICD-11 PTSD, the traumatic events that were more strongly associated with ICD-11 PTSD included sexual assault, unwanted sexual contact, exposure to war, and exposure to severe human suffering; traumatic events that are widely recognised as the most distressing/impairing ${ }^{30,39}$. Furthermore, exposure to severe human suffering was found to significantly increase the likelihood of having a 'unique' ICD-11 PTSD 
diagnosis compared to a 'unique' DSM-5 PTSD diagnosis, whereas exposure to a transportation accident was found to decrease the likelihood of having a 'unique' ICD-11 PTSD diagnosis. Here again evidence was found to suggest that ICD-11 PTSD may be associated with increased scores on an underlying distress continuum.

Several limitations associated with the current study should be recognised. While the representative nature of the current sample is a key strength, the current findings may not generalize to IDP populations in culturally distinct contexts. The use of self-report measures to estimate prevalence and comorbidity rates may be considered sub-optimal as compared to the use of diagnostic interviews. It is possible therefore that estimates of the prevalence and comorbidity may be somewhat overestimated. However, for the purposes of the current study where we sought to compare DSM-5 and ICD-11 prevalence, and their comorbidity with GAD and MDD, this limitation was constant across all analyses meaning that current results are interpretable. Finally, it was not possible to assess comorbidity rates with disorders reflecting the 'Externalizing' and 'Thought Disorder' dimensions of psychopathology. It's possible that differences in comorbidity rates for ICD-11 and DSM-5 could vary depending upon which disorders are selected. Future research should therefore seek to estimate comorbidity with disorders reflecting traditional Externalizing psychopathology (e.g., addiction) and Thought Disorder psychopathology (e.g., schizophrenia).

In conclusion, a fundamental goal of the ICD-11 is that diagnoses be crossculturally relevant, particularly within low-resourced and/or humanitarian crisis situations. The current findings with Ukrainian IDPs provides initial evidence that the ICD-11 diagnosis of PTSD is meaningful in such a context. Our findings have 
important implications for clinicians working with IDPs. The ICD-11 diagnosis of PTSD is particularly helpful at detecting individuals experiencing higher levels of psychiatric distress and disability, and therefore those in the greatest need of clinical intervention. The ICD-11 model may therefore be considered optimal in humanitarian contexts where clinical resources are limited. However, clinicians should also be aware that the ICD-11 criteria will likely yield fewer diagnostic cases of PTSD compared to the DSM-5 criteria and should therefore screen for other psychiatric disorders that are common posttrauma.

\section{References}

1. UNHCR. Global Trends Forced Displacement in 2016 [Available from: http://www.unhcr.org/globaltrends2016/]

2. World Bank Group, WHO, editors. Report of Proceedings of Event "Out of the Shadows: Making Mental Health a Global Development Priority." 2016; Washington, DC: World Bank Group.

3. American Psychiatric Association. Diagnostic and statistical manual of mental disorders, $5^{\text {th }}$ ed. Washington DC: American Psychiatric Association, 2013.

4. Maercker A, Brewin CR, Bryant RA et al. Diagnosis and classification of disorders specifically associated with stress: proposals for ICD-11. World Psychiatry 2013;12:198-206.

5. Galatzer-Levy IR, Bryant RA. 636,120 ways to have posttraumatic stress disorder. Perspect Psychol Sci 2013;8:651-62.

6. O'Donnell ML, Alkemade N, Nickerson A et al. Impact of the diagnostic changes to post-traumatic stress disorder for DSM- 5 and the proposed changes to ICD-11. Br J Psychiatry 2014;205:230-5. doi:

10.1192/bjp.bp.113.135285 
7. Hafstad GS, Thoresen S, Wentzel-Larsen T, Maercker A, Dyb G. PTSD or not PTSD? Comparing the proposed ICD-11 and the DSM-5 PTSD criteria among young survivors of the 2011 Norway attacks and their parents. Psychol Med 2017;47:1283-91. doi: 10.1017/S0033291716002968

8. Hyland P, Shevlin M, McNally S, Murphy J, Hansen M, Elklit A. Exploring differences between the ICD-11 and DSM-5 models of PTSD: Does it matter which model is used? J Anxiety Disord 2016;37:48-53. doi:

10.1016/j.janxdis.2015.11.002

9. Wisco BE, Miller MW, Wolf EJ et al. The impact of proposed changes to ICD11 on estimates of PTSD prevalence and comorbidity. Psychiatry Res 2016;240: 226-33. doi: http://dx.doi.org/10.1016/j.psychres.2016.04.043

10. Hansen M, Hyland P, Armour C, Elklit A, Shevlin, M. Less is more? Assessing the validity of the ICD-11 model of PTSD across multiple trauma samples. Eur J Psychotraumatol 2015;6: 28766. doi: http://dx.doi.org/10.3402/ejpt.v6.28766

11. Hyland $P$, Shevlin $M$, Brewin $C R$ et al. Factorial and discriminant validity of ICD-11 PTSD and CPTSD using the new International Trauma Questionnaire. Acta Psychiatr Scand 2017; In Press.

12. Stein DJ, McLaughlin KA, Koenen KC et al. DSM-5 and ICD-11 definitions of posttraumatic stress disorder: investigating "narrow" and "broad" approaches. Depress Anxiety 2014;31:494-505. doi: 10.1002/da.22279.

13. Cloitre M, Roberts NP, Bisson JI, Brewin CR. The International Trauma Questionnaire (ITQ). Unpublished Measure.

14. Karatzias T, Shevlin M, Fyvie C, Hyland P, Efthymiadou E, Wilson D, Roberts N, Bisson Jl, Brewin CR, Cloitre M. An initial psychometric assessment of an ICD-11 based measure of PTSD and complex PTSD (ICD-TQ): Evidence of 
construct validity. J Anxiety Disord 2016;44:73-9. doi:

http://dx.doi.org/10.1016/j.janxdis.2016.10.009

15. Murphy S, Elklit A, Dokkedahl S, Shevlin M. Testing the validity of the proposed ICD-11 PTSD and complex PTSD criteria using a sample from Northern Uganda. Eur J Psychotraumatol 2015;7:32678. doi: http://dx.doi.org/10.3402/ejpt.v7.32678

16. Roberts B, Makhashvili N, Javakhishvili J, Karachevsky A, Kharchenko N, Shpiker M, Richardson E. Mental health care utilisation among internally displaced persons in Ukraine: results from a nation-wide survey. Epidemiol Psychiatr Sci 2017; In Press.

17. Fisher RH, Ick T, Grasso M, Vaudrey J, McFarland W. Resource Guide: Time Location Sampling (TLS). San Francisco: San Francisco Department of Public Health, 2007.

18. Deng F. Guiding principles on internal displacement. New York: United Nations, 1998.

19. Weathers FW, Blake DD, Schnurr PP, Kaloupek DG, Marx BP, Keane TM. The PTSD Checklist for DSM-5 (PCL-5). Instrument available from the National Center for PTSD at www.ptsd.va.gov

20. Bovin MJ, Marx BP, Weathers FW, Gallagher MW, Rodriguez P, Schnurr PP, Keane TM. Psychometric properties of the PTSD Checklist for Diagnostic and Statistical Manual of Mental Disorders-Fifth Edition (PCL-5) in veterans.

Psychol Assess 2016;28:1379-91. doi: 10.1037/pas0000254

21. Weathers FW, Blake DD, Schnurr PP, Kaloupek DG, Marx BP, Keane TM. The Life Events Checklist for DSM-5 (LEC-5), 2013. Instrument available from the National Center for PTSD at www.ptsd.va.gov 
22. Spitzer RL, Kroenke K, Williams JB, Lowe B. A brief measure for assessing generalized anxiety disorder: the GAD-7. Arch Intern Med 2006;166:1092-7. doi: 10.1001/archinte.166.10.1092

23. Kroenke K, Spitzer RL, Williams JB, Lowe B. The Patient Health Questionnaire Somatic, Anxiety, and Depressive Symptom Scales: a systematic review. Gen Hosp Psychiatry 2010;32:345-59. doi: 10.1016/j.genhosppsych.2010.03.006.

24. Kroenke K, Spitzer RL, Williams JBW. The PHQ-9: Validity of a brief depression severity measure. J Gen Intern Med 2001;16:606-13. doi: 10.1046/j.1525-1497.2001.016009606.x

25. Manea L, Gilbody S, McMillan D. A diagnostic meta-analysis of the Patient Health Questionnaire-9 (PHQ-9) algorithm scoring method as a screen for depression. Gen Hosp Psychiatry 2015;37:67-75. doi:

10.1016/j.genhosppsych.2014.09.009

26. World Health Organization. World Health Organization Disability Assessment Schedule II, 2001. Available at: www.who.int/icidh/whodas/. Accessed on: 27 April, 2017.

27. Andrews G, Kemp A, Sunderland M, Von Korff M, Ustun TB. Normative data for the 12 item WHO Disability Assessment Schedule 2.0. PLoS One 2009;4:e8343. Doi: https://doi.org/10.1371/journal.pone.0008343

28. Van Ommeren M, Sharma B, Thapa S, Makaju R, Prasain D, Bhattarai R, De Jong J. Preparing instruments for transcultural research: Use of the translation monitoring form with Nepali-speaking Bhutanese refugees. Transcultural Psychiatry 1999;36:285-301. 
29. Cohen J. A Coefficient of Agreement for Nominal Scales. Educ Psychol Meas 1960: 20:37-46.

http://dx.doi.org/10.1177/001316446002000104

30. McHugh ML. Interrater reliability: the kappa statistic. Biochemia Medica. 2012;22(3):276-282.

31. Tolin DF, Foa EB. Sex differences in trauma and posttraumatic stress disorder: A quantitative review of 25 years of research. Psychol Bull 2006;132:959-92.

32. Kessler RC, Berglund P, Delmer O, Jin R, Merikangas KR, Walters EE. Lifetime prevalence and age-of-onset distributions of DSM-IV disorders in the National Comorbidity Survey Replication. Arch Gen Psychiatry 2005;62:593602.

33. Bisson Jl. What happened to harmonization of the PTSD diagnosis? The divergence of ICD11 and DSM5. Epidemiol Psychiatr Sci 2013;22:205-7. doi: $10.1017 / S 2045796013000164$

34. Kotov R, Krueger RF, Watson D et al. The Hierarchical Taxonomy of Psychopathology (HiTOP): A Dimensional Alternative to Traditional Nosologies. Journal of Abnormal Psychology, 2017;126:454-77. Doi: http://dx.doi.org/10.1037/abn0000258

35. Markon KE, Krueger RF, Watson D. Delineating the structure of normal and abnormal personality: An integrative hierarchical approach. J Pers Soc Psychol 2005;88:139-157. Doi: http://dx.doi.org/10.1037/0022-3514.88.1.139

36. Kotov R, Ruggero CJ, Krueger RF, Watson D, Yuan Q, Zimmerman M. New dimensions in the quantitative classification of mental illness. Arch Gen 
Psychiatry 2011;68:1003-11. Doi:

http://dx.doi.org/10.1001/archgenpsychiatry.2011.107

37. Caspi A, Houts RM, Belsky, DW. The p factor: One general psychopathology factor in the structure of psychiatric disorders? Clin Psychol Sci 2014;2:119_ 137. Doi: http://dx.doi.org/10.1177/2167702613497473

38. Bollen K, Lennox R. Conventional wisdom on measurement: A structural equation perspective. Psychol Bull 1991;110:305-14.

39. Hyland $\mathrm{P}$, Murphy $\mathrm{J}$, Shevlin $\mathrm{M}$ et al. Variation in post-traumatic response: The role of trauma type in predicting ICD-11 PTSD and CPTSD symptoms. Soc Psychiatry Psychiatr Epidemiol 2017;52:727-736. doi: 10.1007/s00127017-1350-8. 
Table 1. Probable PTSD Prevalence based on DSM-5 and ICD-11.

\begin{tabular}{|c|c|c|c|c|}
\hline PTSD diagnosis & Male & Female & Total & $\chi^{2}(d f) p$ \\
\hline & $\mathrm{N}=677$ & $\mathrm{~N}=1423$ & $\mathrm{~N}=2100$ & \\
\hline DSM-5 & $133(23.1 \%)$ & $442(31.1 \%)$ & $575(27.4 \%)$ & $30.07(1)<.001$ \\
\hline ICD-11 & $87(13.1 \%)$ & $350(24.6 \%)$ & $438(21.0 \%)$ & $36.30(1)<.001$ \\
\hline
\end{tabular}

Note: $\chi^{2}=$ chi-square test, $\mathrm{df}=$ degrees of freedom, $\mathrm{p}=$ level of statistical significance. 
Table 2. Comorbidity of DSM-5 and ICD-11 PTSD with Generalized Anxiety Disorder

\begin{tabular}{|l|c|c|c|}
\hline PTSD diagnosis & $\%$ with GAD & $\%$ with MDD & $\begin{array}{c}\% \text { with GAD and } \\
\text { MDD }\end{array}$ \\
\hline \multicolumn{3}{|c|}{ Comorbidity rates for ICD-11 and DSM-5 PTSD } \\
\hline ICD-11 & $54.6 \%$ & $64.3 \%$ & $43.2 \%$ \\
\hline DSM-5 & $46.7 \%$ & $57.7 \%$ & $35.0 \%$ \\
\hline Difference & $7.9 \%^{*}$ & $6.5 \%^{*}$ & $8.1 \%{ }^{*}$ \\
\hline \multicolumn{2}{|c|}{ Comorbidity rates for 'unique' cases of ICD-11 and DSM-5 PTSD } \\
\hline Unique ICD-11 & $29.3 \%$ & $34.6 \%$ & $22.8 \%$ \\
\hline Unique DSM-5 & $25.5 \%$ & $36.6 \%$ & $15.9 \%$ \\
\hline Difference & $3.8 \%{ }^{*}$ & $2.0 \%{ }^{*}$ & $6.9 \%{ }^{*}$ \\
\hline
\end{tabular}

Note: GAD = Generalised Anxiety Disorder; MDD= Major Depressive Disorder; * all differences are statistically significant $(p<.05)$. 
Table 3. Association Between Trauma Exposure and Probable DSM-5 and ICD-11 PTSD.

\begin{tabular}{|c|c|c|c|c|}
\hline & $N(\%)$ exposed & DSM-5 PTSD & ICD-11 PTSD & $\begin{array}{l}\text { Unique ICD-11 } \\
\text { PTSD }^{\mathrm{a}}\end{array}$ \\
\hline Life Event & & OR $(95 \% \mathrm{Cl})$ & OR $(95 \% \mathrm{Cl})$ & OR $(95 \% \mathrm{Cl})$ \\
\hline $\begin{array}{l}\text { 1. Natural disaster (for example, flood, } \\
\text { hurricane, tornado, earthquake) }\end{array}$ & $98(4.5 \%)$ & $2.06(1.36-3.11)^{*}$ & $1.59(1.01-2.50)^{*}$ & $.72(.25-2.06)$ \\
\hline 2. Fire or explosion & $637(29.0 \%)$ & $1.39(1.13-1.70)^{*}$ & $1.68(1.35-2.10)^{*}$ & $1.19(.66-2.15)$ \\
\hline $\begin{array}{l}\text { 3. Transportation accident (for example, } \\
\text { car accident, boat accident, train } \\
\text { wreck, plane crash) }\end{array}$ & $204(9.3 \%)$ & $1.87(1.38-2.54)^{*}$ & $1.50(1.07-2.10)^{*}$ & $.22(.06-.85)^{*}$ \\
\hline $\begin{array}{l}\text { 4. Serious accident at work, home, or } \\
\text { during recreational activity }\end{array}$ & $286(13.0 \%)$ & $1.86(1.43-2.42)^{*}$ & $1.43(1.07-1.91)^{*}$ & $.45(.19-1.05)$ \\
\hline 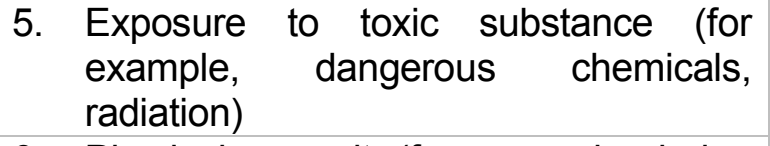 & $64(2.9 \%)$ & $1.82(1.09-3.06)^{*}$ & $1.21(.67-2.18)$ & $.49(.11-2.08)$ \\
\hline $\begin{array}{l}\text { 6. Physical assault (for example, being } \\
\text { attacked, hit, slapped, kicked, beaten } \\
\text { up) }\end{array}$ & $261(11.9 \%)$ & $2.38(1.82-3.12)^{*}$ & $2.04(1.53-2.73)^{*}$ & $.60(.26-1.38)$ \\
\hline $\begin{array}{l}\text { 7. Assault with a weapon (for example, } \\
\text { being shot, stabbed, threatened with a } \\
\text { knife, gun, bomb) }\end{array}$ & $272(12.4 \%)$ & $1.56(1.19-2.05)^{*}$ & $1.73(1.30-2.31)^{*}$ & $1.42(.67-2.99)$ \\
\hline $\begin{array}{l}\text { 8. Sexual assault (rape, attempted rape, } \\
\text { made to perform any type of sexual act } \\
\text { through force or threat of harm) }\end{array}$ & $54(2.5)$ & $2.95(1.71-5.09)^{*}$ & $3.66(2.12-6.32)^{*}$ & $1.789 .43-7.31)$ \\
\hline $\begin{array}{l}\text { 9. Other unwanted or uncomfortable } \\
\text { sexual experience }\end{array}$ & $49(2.2 \%)$ & $2.07(1.15-3.75)^{*}$ & $3.28(1.82-5.92)^{*}$ & $4.54(.73-28.25)$ \\
\hline $\begin{array}{l}\text { 10. Combat or exposure to a war-zone (in } \\
\text { the military or as a civilian) }\end{array}$ & $1566(71.3 \%)$ & $.97(.79-1.20)$ & $1.28(1.01-1.63)^{*}$ & $1.36(.76-2.43)$ \\
\hline $\begin{array}{l}\text { 11. Captivity (for example, being } \\
\text { kidnapped, abducted, held hostage, } \\
\text { prisoner of war) }\end{array}$ & $43(2.0 \%)$ & $1.15(.59-2.22)$ & $1.30(.65-2.61)$ & $.22(.00-48.26)$ \\
\hline
\end{tabular}


DSM-5 and ICD-11 PTSD 31

\begin{tabular}{|c|c|c|c|c|}
\hline 12. Life-threatening illness or injury & $353(16.1 \%)$ & $2.59(2.04-3.30)^{\star}$ & $2.49(1.93-3.21)^{*}$ & $.87(.46-1.67)$ \\
\hline 13. Severe human suffering & $929(42.3)$ & $2.22(1.83-2.70)^{*}$ & $2.69(2.16-3.34)^{*}$ & $1.76(1.01-3.08)^{*}$ \\
\hline $\begin{array}{l}\text { 14. Witnessed sudden violent death (for } \\
\text { example, homicide, suicide) }\end{array}$ & $147(6.7 \%)$ & $1.75(1.23-2.47)^{\star}$ & $1.54(1.05-2.26)^{*}$ & $.62(.19-1.97)$ \\
\hline 15. Witnessed sudden accidental death & 121 (5.5\%) & $1.61(1.10-2.36)^{*}$ & $1.59(1.05-2.40)^{*}$ & $.62(.16-2.38)$ \\
\hline $\begin{array}{l}\text { 16. Serious injury, harm, or death you } \\
\text { caused to someone else }\end{array}$ & $40(1.8 \%)$ & $3.00(1.60-5.62)^{*}$ & $2.80(1.46-5.38)^{*}$ & $1.19(.33-4.32)$ \\
\hline
\end{tabular}

Note: OR $(95 \% \mathrm{Cl})=$ Odds ratio with $95 \%$ confidence intervals; ${ }^{*}=p<.05{ }^{\text {a }}$ reference group in this case are the 'unique' DSM-5 PTSD cases. 